\title{
EDITORIAL
}

\section{Mobile and contextual learning}

Is mobile learning just a part of everyday learning? This is a relevant question in an age when most people throughout the world now have access to mobile phones and mobility is increasingly taken for granted. In one sense, mobile learning is no different to carrying a textbook or learning through conversations at home, as part of formal education or in the workplace. The technology may be more engaging, but is the learning any different? Despite the ubiquity of mobile phones, smartphones, mp3 players and, increasingly, access to Wi-Fi connections and GPS navigation, the reasons for using mobile and wireless technologies in education are not yet widely known. There is significant specialist expertise, built from ten years of research (and more recently, reflective practice) to demonstrate the unique characteristics of mobile learning, which include orchestrating shared learning with personal devices across formal and informal settings, providing immediately useful information, offering timely revision and reflection, connecting real and virtual locations, and enriching field trips and cultural visits. This knowledge needs to be disseminated and examined from new perspectives.

Learning when mobile means that context becomes all-important, since even a simple change of location is an invitation to revisit learning, in both a literal sense (to apply it, reflect on it, reinforce it, share it) and metaphorical, to reconsider what constitutes learning or what makes it effective in a given situation. Although 'mobile' and 'contextual' learning are almost synonymous, the title of this special issue reflects a substantial overlap rather than a single identity. It invites a reflection on the nature of context in learning. When the setting for learning is a classroom or lecture theatre, then context can be seen as a set of circumstances and resources that enable and support prescribed events such as lessons or lectures. If we now broaden this to include learning that occurs across settings, through distance communication, or between real and virtual worlds, then context becomes a continually unfolding property of the interactions between people and their goals, settings and technologies. Context cannot be easily predicted in advance, but is shaped from minute to minute by mobile learners and their devices.

The paper by Cornelius and Marston adds to our understanding by explaining how simulation activities using SMS can create a 'virtual context' as learners are transported into a world existing in parallel with their physical and social experience. The authors comment on 'presence' and 'disruption', currently popular themes that other authors in this issue have also explored.

Taking account of context also implies understanding the lifestyles of contemporary learners and the demands of new curricula, which are rapidly evolving to include a range of student commitments such as paid or voluntary work and practical experience in combination with study. In these respects, as in others, there will be variation between disciplines, as noted in the paper by Percival and Percival, arguing that 
different mobile learning models are needed for technical and liberal arts programmes of study. These authors recommend the integration of technology with the students' educational experience, another major theme echoed by other authors in this issue.

The biology students in the study by Pfeiffer et al. were supported by visual materials on portable DVD players on their trips to identify fish species in the sea. This situated learning scenario was the setting for a comparison of static and dynamic visualisations as learning resources. Here, learning is contextualised through a practical real-world experience and the availability of the most effective learning materials in the location where they are most needed. The DVD players could not be used in the water, only on the seashore, but this apparent constraint also helped to delineate direct observation from the activity of determining fish species, whilst still keeping them close together physically and in time.

Jones, Edwards and Reid take the problem of transition to higher education, looking at how Coach Education and Sports Development students, who have many sports commitments alongside their studies, could be alerted to and engaged with learning tasks and deadlines located in the institutional Virtual Learning Environment (VLE), through use of SMS and a texting management service. The learning design includes face-to-face sessions followed by texts being sent to students, activities in the VLE, and further texts to students, with pre-session activities completing an integrated weekly learning experience.

Another successful learning design is presented by Sutton-Brady et al. who trialled the use of short-format podcasts with Commerce students. The podcasts were focused on delivering information to support assessment. Delivery of key information, which would otherwise need to be repeated, became more streamlined, whilst the students perceived the information as being more personalised: a familiar voice in the ear. Since the students involved in this study were not particularly mobile, they tended to listen to podcasts on their computers rather than mobile devices; therefore the mobility here consists in extending the lecturer's personal 'presence' and creating more vivid contexts for learning beyond the classroom. Much like Jones, Edwards and Reid, the authors recommend designing mobile activities as pre-class and post-class sessions, which again suggests that an integrated approach is most likely to be successful.

To effect such integration, teachers need to have a good understanding of what mobile and contextual learning can offer. What better way to achieve such understanding than to use mobile technologies for one's own professional development? The paper by Aubusson, Schuck and Burden considers how mobile learning can support reflection and collaboration in teachers' professional learning, as well as providing an insight into how the shared use of mobile devices by teachers and students adds value to learning by both parties. The idea that mobile technology can be a catalyst for new learning conversations is made evident here and should continue to inspire further research.

Agnes Kukulska-Hulme The Open University, UK Mike Sharples University of Notthingham, UK 